NBER WORKING PAPER SERIES

\title{
GAMES AND RESOURCES
}

Bård Harstad

Matti Liski

Working Paper 18519

http://www.nber.org/papers/w18519

\section{NATIONAL BUREAU OF ECONOMIC RESEARCH \\ 1050 Massachusetts Avenue \\ Cambridge, MA 02138 \\ November 2012}

This article is prepared as Ch. 154 in the forthcoming Encyclopedia of Energy, Natural Resources and Environmental Economics, Elsevier (Ed: Jason Shogren). The views expressed herein are those of the authors and do not necessarily reflect the views of the National Bureau of Economic Research.

NBER working papers are circulated for discussion and comment purposes. They have not been peerreviewed or been subject to the review by the NBER Board of Directors that accompanies official NBER publications.

(C) 2012 by Bård Harstad and Matti Liski. All rights reserved. Short sections of text, not to exceed two paragraphs, may be quoted without explicit permission provided that full credit, including @ notice, is given to the source. 
Games and Resources

Bård Harstad and Matti Liski

NBER Working Paper No. 18519

November 2012

JEL No. C7,H7,Q0

\begin{abstract}
$\underline{\text { ABSTRACT }}$
This article presents a sequence of simple and related models to analyze the strategic use of natural resources. Game theory is the natural tool for such an analysis, whether the resource is private or publicly owned, whether it is renewable or exhaustible, whether the game is static or dynamic, and whether or not the users can strategically invest in technologies. Equilibrium extraction is too large and comes too early for public resources, but the opposite is true for private resources. The effects add up nicely when the resource has both private and public-good aspects.
\end{abstract}

\author{
Bård Harstad \\ Kellogg School of Management \\ Northwestern University \\ 2001 Sheridan Road \\ Evanston, IL 60208 \\ and NBER \\ harstad@kellogg.northwestern.edu \\ Matti Liski \\ Aalto University School of Economics \\ Helsinki \\ matti.liski@aalto.fi
}




\section{Introduction}

Game theory is the natural tool when analyzing the strategic use of natural resources. This article presents a sequence of related simple two-stage games to illustrate a range of problems, generating a large number of lessons. We study environments that are static as well as dynamic; resources that are publicly owned as well as privately owned; strategic investments in substitute (or green) technology as well as complementary technology (such as extraction technology). The lessons vary greatly across the institutional settings, but the lessons have in common that they are all derived from the use of simple game theory. This way, the article provide a survey of how game theory can be fruitfully employed when studying environmental and resource economics. The resource itself can be, for instance, fish, forests, fossil fuel, fresh air, or fresh-water lakes, to mention examples starting with the letter $f$.

We start by studying the common-pool problem, assuming that the resource is publicly owned. Each user is then extracting too much, ignoring the negative externality on the other users. Still, efficiency is further reduced in a simple dynamic common-pool problem with two stages: Each user then has an incentive to extract a lot at the first stage, since this will discourage the other users from extracting at the second stage. The outcome is that even more of the resource is extracted. Furthermore, the intertemporal allocation of the extracted amounts is also inefficient: too much is extracted in the beginning, and too little later on (conditional on the total amount extracted). These negative results continue to hold if the users can invest in substitute technologies, such as abatement technology or renewable energy sources. Each user then has an incentive to invest strategically little, as a visible and credible commitment to extract more of the resource at the second stage. This is beneficial to the investor, since the other users will then find it optimal to extract less. For the same reason, the users prefer to invest a lot in complementary technologies, such as extraction technologies, since this, too, will effectively commit an investor to extract a lot and thus discourage the other users from extracting.

Our third section finds that, if the resource is privately owned, the main results are reversed. While privatization solves the common-pool problem, concentrated ownership generates market power that will be exploited. As a result, the seller prefers to extract strategically little in order to raise the price, or the buyer buys strategically little to reduce the price. Also the intertemporal allocation of extraction rates is inefficient, and it becomes too conservative. The owner prefers to conserve the resource in order to raise the price today, to discourage competitors from producing in the future, as well as to discourage buyers from investing in substitute technologies that could reduce their willingness to pay in the resource.

The fourth section lets the resource have private as well as public-good aspects. The

owner decides how much to extract, but extraction generates a negative externality. This is the natural model when analyzing global warming, for example. If a country, or a coalition of countries, implements a policy consisting of quotas or taxes, then the equilibrium policy is going to reflect the public-good aspect (i.e., the environmental harm) as well as the private aspect (i.e., the coalition prefers a policy improving its terms-oftrade). The outcome is, again, far from efficient. 


\section{Public Resources}

This section presents a very simple model of common resources. However, after describing the model, we will argue that it can capture quite complicated situations. This will be demonstrated throughout the paper as we, progressively, analyze static commonpool problems, dynamic common-pool problems, strategic situations with technological choices, and private resources. We start with a perfectly symmetric model (asymmetries are discussed in the next section, where the resource can be privately owned).

The resource stock is measured by $s \in(0, \infty)$ and the set of users is $I=\{1,2, \ldots, n\}$. Each user, $i \in I$, decides to extract $z_{i}$ of the resource. Such extraction may harm the other users, creating a negative externality. This externality arises because $i$ 's extraction depletes the resource stock, and each user's valuation of the stock is $v(s)$. In particular, $i$ 's utility is:

$$
\begin{aligned}
U\left(x_{i}, z_{i}, s\right) & =u\left(x_{i}, z_{i}\right)+v(s), \text { and } \\
s & =s_{0}-\sum_{I} z_{i} .
\end{aligned}
$$

So, $s_{0}$ is the initial level of the resource. Variable $x_{i}$ represents other decisions made by user $i$, assumed to not generate any direct externalities. It can be interpreted as a policy parameter or a technology choice. We assume $u(\cdot)$ is concave in $x_{i}$ and that $\arg \max _{x_{i}} u\left(x_{i}, z_{i}\right)+v(s)$ is always interior.

Note that many applications fit the functional forms (1)-(2). For example, $s$ may represent the stock of clean air if $z_{i}$ is $i$ 's pollution level, reducing this stock. The environmental harm from pollution is then given by the convex function $c\left(\sum_{I} z_{i}\right) \equiv$ $v\left(s_{0}\right)-v\left(s_{0}-\sum_{I} z_{i}\right)$, where $v\left(s_{0}\right)$ is simply a constant, and it can be ignored without loss of generality.

A technical note: The functional forms (1)-(2) can capture the essence of a wide class of resource problems, even settings that are dynamic and without the additive structure as in (1). In dynamic settings, more extraction today leads to a lower amount of resource for the future. But even then, we can typically write $i$ 's payoff as $U_{t}\left(x_{i, t}, z_{i, t}, s_{t-1}\right)=$ $\widetilde{u}\left(x_{i, t}, z_{i, t}, f\left(s_{t-1}\right)\right)+\delta \widetilde{v}\left(f\left(s_{t-1}\right)-\sum_{I} z_{i, t}\right)$, where $f(\cdot)$ is the growth-function of the resource, and the continuation value, starting in the subsequent period, is captured by $v(s) \equiv \delta \widetilde{v}\left(f\left(s_{t-1}\right)-\sum_{I} z_{i, t}\right)$. In Markov-perfect equilibria, the continuation value will depend only the stock, exactly as captured by (1)-(2). Consequently, most symmetric settings with common resources can be formalized by the model (1)-(2), even if they are dynamic and not necessarily additive in the dynamic setting. In fully dynamic models, $v(\cdot)$ is endogenized. In this article, we simply take $v(\cdot)$ as exogenously given to further analyze simpler one and two stage games.

\subsection{The Static Common Pool Problem}

For simplicity, we refer to the first-best as the outcome preferred by a social planner maximizing the sum of the users' payoffs.

Definition 1. The first-best is an allocation of $\left\{x_{i}, z_{i}\right\}_{i \in I}$ maximizing the sum of 
utilities:

$$
\begin{aligned}
\max _{\left\{x_{i}\right\},\left\{z_{i}\right\}} & \sum_{I} U\left(x_{i}, z_{i}, s\right) \\
\frac{\partial u\left(x_{i}, z_{i}\right)}{\partial z_{i}} & =n \frac{\partial v(s)}{\partial s} \text { and } \frac{\partial u\left(x_{i}, z_{i}\right)}{\partial x_{i}}=0 .
\end{aligned}
$$

The second-order conditions for the planner's problem are satisfied when $\left|u_{12}\right| \leq \sqrt{u_{11}\left(u_{22}+n^{2} v^{\prime \prime}\right)}$ since $\partial^{2} u\left(x_{i}, z_{i}\right) / \partial q_{i}^{2}+n^{2} v^{\prime \prime}<0$ and $\partial^{2} u\left(x_{i}, z_{i}\right) / \partial x_{i}^{2}<0$.

However, in the game referred to as the problem of the commons, the variables are not chosen by a social planner, but by the individual users. When all the users act simultaneously, the natural equilibrium concept is Nash equlibrium.

Definition 2. A Nash equilibrium is a set of choices, $\left\{x_{i}, z_{i}\right\}_{i \in I}$, such that, when user $i$ takes as given the other users' choices, $\left\{x_{j}, z_{j}\right\}_{j \in I \backslash i}$, then $\left\{x_{i}, z_{i}\right\}$ maximizes $U\left(x_{i}, z_{i}, s\right)$.

We use superscript $s$ to denote the static version of the game, where all users act simultaneously and only once.

Proposition 1 Consider the static common-pool problem: (i) Each user's extraction $z_{i}^{s}$ is larger than the first-best:

$$
\frac{\partial u\left(x_{i}, z_{i}^{s}\right)}{\partial z_{i}^{s}}=v^{\prime}(s)<n v^{\prime}(s) .
$$

(ii) For any given $z_{i}$, the equilibrium $x_{i}^{s}$ is socially optimal:

$$
\frac{\partial u\left(x_{i}^{s}, z_{i}\right)}{\partial x_{i}^{s}}=0 .
$$

Since user $i$ does not internalize the negative externality on the other users, $i$ extracts too much. In other words, $i$ extracts more from the common resource than what a social planner would prefer and as specified by the first-best. Note that the difference between the equilibrium extraction and the first-best increases in the number of users, $n$. Since choice-variable $x_{i}$ was assumed to create no externality, it is optimally chosen. This fact is natural in the static setting but it might no longer hold in dynamic settings, as we soon will learn.

In principle, $v(\cdot)$ can be concave or convex. If $v(\cdot)$ were convex, extraction levels are strategic complements, in the sense that $i$ benefits more from increasing $z_{i}$ if $z_{j}, j \neq i$, large:

$$
\frac{\partial^{2} U}{\partial z_{i} \partial z_{j}}>0 \Leftrightarrow v^{\prime \prime}(s)>0 .
$$

In this situation, user $i$ prefers to extract more if the other users are expected to extract a lot. A convex $v(\cdot)$ may be reasonable for resources close to extinction or depletion, for example, where reducing extraction levels is beneficial for one user only if also the other users attempt to conserve the resource. The second-order condition, when choosing $z_{i}$, may then not hold, and, in fact, it never holds if $u(\cdot)$ is linear in $z_{i}$. When the second-order 
condition fails, the consequence is multiple equilibria, where user $i$ 's optimal extraction level $z_{i}$ is large (small) if the other users are believed to extract a lot (little). The extraction game is then a coordination game.

If $v(\cdot)$ is concave, instead, extraction levels are strategic substitutes, in the sense that $i$ benefits more from increasing $z_{i}$ if $z_{j}, j \neq i$, is small:

$$
\frac{\partial^{2} U}{\partial z_{i} \partial z_{j}}<0 \Leftrightarrow v^{\prime \prime}(s)<0 .
$$

One user is then preferring to extract less if another user is expected to extract a lot. This may be reasonable for most resources. Fish-stocks, for example, typically have smaller growth-rates at high levels, contributing to a concave $v(\cdot)$. The harm from pollution is typically assumed to be an increasing and convex function, implying that the utility of less pollution, or clean air, is an increasing and concave function. Since the second-order conditions tend to hold, in this case, the equilibrium is unique. The rest of the section presumes that $v(\cdot)$ is concave.

\subsection{A Dynamic Common Pool Problem}

The above simple model can illustrate the forces at play also in simple dynamic common pool problems. One interpretation of variable $x_{i}$ is that it measures user $i$ 's early extraction level. Suppose there are two stages: $i$ first extracts $x_{i}$ for the first stage and $y_{i}$ for the second stage. The total extraction level is $z_{i} \equiv x_{i}+y_{i}$, as before. Suppose consumption equals extraction and generates the utility $w^{t}(\cdot), t \in\{1,2\}$, where $w^{t}(\cdot)$ is assumed to be increasing and concave. Then, user $i$ 's utility $u(\cdot)$ over the two stages can be written as:

$$
u\left(x_{i}, z_{i}\right)=w^{1}\left(x_{i}\right)+w^{2}\left(z_{i}-x_{i}\right) .
$$

In the static or normal-form version of this game, user $i$ will simply set both $x_{i}$ and $z_{i}$ at the same time. In that case, it follows from Proposition 1 that:

$$
w^{1 \prime}\left(x_{i}\right)=w^{2 \prime}\left(z_{i}-x_{i}\right)=v^{\prime} .
$$

Hence, given user $i$ 's total extraction $z_{i}$, $i$ 's extraction in period 1 is efficient. In other words, the intertemporal allocation of the extracted amount is efficient even if the total amount itself is suboptimally large.

However, if the users cannot commit, they will choose the extraction levels for the two states sequentially. The first-stage extraction $x_{i}$ is chosen, and observed by everyone, before the users choose the second-stage extraction, $z_{i}-x_{i}$. With this dynamic or twostage extensive form game, it is natural to limit attention to subgame-perfect equilibria. That is, we will require that the strategies at stage two continue to constitute a Nash equilibrium in the game that is played at that stage, no matter what the first-stage actions turned out to be. Since the $x_{i} \mathrm{~s}$ are observed before the $z_{i}$ s are set, the latter choices are likely going to be functions of the set of $x_{i}$-choices. That is, $z_{i}=z_{i}(x)$, where $x=\left(x_{1}, \ldots, x_{n}\right)$.

Definition 3: The strategies $\left(x_{i}, z_{i}(x)\right)_{i \in I}$ constitute a subgame-perfect equilibrium if they constitute a Nash equilibrium and if also $\left(z_{i}(x)\right)_{i \in I}$ constitute a Nash equilibrium at the second stage for every feasible $x=\left(x_{1}, \ldots, x_{n}\right)$. 
In our two-stage model, the total payoff for $i$ from choosing $x_{i}$, given $x_{-i}$, is

$$
u\left(x_{i}, z_{i}\left(x_{i}, x_{-i}\right)\right)+v\left(s_{0}-\sum_{j \in I} z_{j}\left(x_{i}, x_{-i}\right)\right) .
$$

Player $i$ can thus anticipate how its first-stage action $x_{i}$ influences extraction not only by $i$ but also by every other $j$. We use superscript $d$ to distinguish the dynamic game from the static. There is a unique subgame-perfect equilibrium.

Proposition 2 Consider the dynamic common-pool problem:

(i) User $i$ 's total extraction $z_{i}^{d}$ is larger than the first-best.

(ii) Given $z_{i}^{d}$, first-period extraction is too large and given by:

$$
w^{1 \prime}\left(x_{i}\right)=\left(1-\frac{(n-1) v^{\prime} v^{\prime \prime}}{n v^{\prime \prime}+w^{2 \prime \prime}}\right) v^{\prime}<v^{\prime} .
$$

(iii) Consequently, both $x_{i}$ and $z_{i}$ are larger than in the static version of the commonpool problem, while every payoff $U_{i}$ is smaller:

$$
z_{i}^{d} \geq z_{i}^{s}, s^{d} \leq s^{s}, \text { and } U_{i}^{d} \leq U_{i}^{s} .
$$

All inequalities are strict as long as $v^{\prime \prime}<0$.

Proof: The proof is ommitted as it would simply be a special case of the next proof. $Q E D$

In other words, each user extracts more when the other users can observe early extraction and thereafter modify their next extraction rates. Since $v$ is concave, more extraction today induces the other users to extract less tomorrow, since the marginal cost of extracting more, $v^{\prime}\left(s_{0}-\sum_{I} x_{i}\right)$, is larger if $x_{i}$ is large. Anticipating this, each user extracts more than in a similar static game.

Note that (3) implies that equilibrium extraction is more aggressive compared to the optimum if $n$ is large and $v$ very concave compared to the concavity of $w^{2}(\cdot)$ (i.e., if $v^{\prime \prime} / w^{2 \prime \prime}$ is large). If $v$ were linear, there would be no such distortion and $x_{i}$ would be first-best, given $z_{i}$. For any strictly concave $v(\cdot)$, however, the dynamic common pool problem is strictly worse than the static version of the game. If $v(\cdot)$ were convex, the opposite would be true: user $i$ would then want to extract little at stage one, since this would raise the benefit for the other users to conserve the resource at stage two.

If the resource $s$ is interpreted as clean air and $z_{i}$ as pollution, then the corollary is simply that each polluter emits more today in order to induce the other users to pollute less in the future. The consequence is more aggregate pollution. If the resource is the stock of fish, each fisherman fishes more than the first-best - not only because he fails to take the static externality into account - but also because more extraction today implies that the other fishermen find it optimal to fish less tomorrow. If the resource-stock grows between the two stages, the model should be slightly modified but the main results would continue to hold. 


\subsection{Technology and Commitment}

The rather general lesson from the previous subsection is that when $v(\cdot)$ is concave, implying that the extraction rates are strategic substitutes, then each user would benefit if it, somehow, could commit to extract more, since the other users would then find it optimal to extract less. In reality, user $i$ has several opportunities to make such a commitment: any policy set in advance, or any investment in technology, may influence user $i$ 's future preference, and these choices can be captured by the parameter $x_{i}$. Thus, the first-stage action $x_{i}$ does not need to be early extraction. It can be anything that does not, by itself, generate externalities on the other users. We will now refer to $x_{i}$ as a technology. Although $x_{i}$ itself is assumed to not generate any externality, it may influence $i$ 's preferences for extraction at stage two. Anticipating this, the other users may extract less if the technology $x_{i}$ is strategically chosen.

Using the similar definition as before, we will say that the technology is a strategic complement to extraction if $u_{12}>0$ and a strategic substitute if $u_{12}<0$.

Proposition 3 Consider the sequential game where the $x_{i}$ s are chosen before the $z_{i} s$. In the subgame-perfect equilibrium, the first-order condition for $x_{i}$ is:

$$
\frac{\partial u}{\partial x_{i}}=\frac{(n-1) v^{\prime} u_{12} / u_{22}}{n+u_{22} / v^{\prime \prime}} .
$$

(i) Thus, if $x_{i}$ is a complement to $z_{i}$ (i.e., if $u_{12}>0$ ), then $x_{i}^{d}>x_{i}^{s}$. If $x_{i}$ is a substitute to $z_{i}$ (i.e., if $u_{12}<0$ ), then $x_{i}^{d}<x_{i}^{s}$.

(ii) Sequential moves worsen the common-pool problem: $z_{i}^{d} \geq z_{i}^{s}, s^{d} \leq s^{s}$, and $U_{i}^{d} \leq U_{i}^{s}$. All inequalities are strict as long as $u_{12} \neq 0$.

Proof: Anticipating all the effects from setting $x_{i}$, the first-order condition is

$$
\begin{aligned}
u_{1}+u_{2} \frac{d z_{i}}{d x_{i}}-v^{\prime}\left(\frac{d z_{i}}{d x_{i}}+(n-1) \frac{d z_{j}}{d x_{i}}\right) & =0 \Rightarrow \\
u_{1}-v^{\prime}(n-1) \frac{d z_{j}}{d x_{i}} & =0 .
\end{aligned}
$$

The strategic effect $\frac{d z_{j}}{d x_{i}}$ can be evaluated by totally differentiating all second-stage firstorder conditions (for $i$ and $j \neq i$ ). Consider thus the impact of a small increase in $x_{i}$ of size $d x_{i}$ (keeping ever other $x_{j}$ constant):

$$
\begin{aligned}
u_{12} d x_{i}+u_{22} d z_{i} & =-v^{\prime \prime} \sum_{l \in I} d z_{l}, \\
u_{22} d z_{j} & =-v^{\prime \prime} \sum_{l \in I} d z_{l} \Rightarrow \\
d z_{j} & =\frac{-v^{\prime \prime}}{\left(u_{22}+v^{\prime \prime}(n-1)\right)} d z_{i} .
\end{aligned}
$$

Substituting (8) into (7), we can, after a few steps, obtain

$$
\frac{d z_{j}}{d x_{i}}=\frac{u_{12} / u_{22}}{n+u_{22} / v^{\prime \prime}}<0 \text { if } u_{12}>0,
$$


while, from (8), we know that $d z_{i} / d x_{i}$ and $d z_{j} / d x_{i}$ have opposite signs. Combining (6) and (9) gives

$$
u_{1}=\frac{(n-1) v^{\prime} u_{12} / u_{22}}{n+u_{22} / v^{\prime \prime}} .
$$

QED

Complementary technologies are, for example, drilling technology, investments in polluting industries, fishing boats, or the breeding of cows for the common grassland. All such investments are larger than what is first-best and, furthermore, they are larger than what the users would have chosen were they not observed and reacted to by the other users of the resource. Since every user invests too much in complementary technology, the result is that extraction is larger, and the resource stock is smaller, than what would be the outcome in a static setting where $x_{i}$ were not chosen strategically before $z_{i}$.

Substitute technologies are, for example, renewable energy-sources, investments in abatement technologies, a district's re-education of fishermen or a gradual exit from the extracting industry. Every such investment level is, in equilibrium, sub-optimally low and, furthermore, it is smaller than the investment levels preferred by the users at the extraction stage (or in a static setting where $x_{i}$ and $z_{i}$ are set simultaneously). Since good substitutes are not invested in to a sufficiently degree, all users will extract too much and more than they would in the similar static game.

As a simple illustration and example, suppose that each user can invest in windmills and consume the generated renewable energy in addition to the energy from fossil fuel. If $x_{i}$ measure the level of renewable energy investment, $i$ enjoys the utility $u\left(x_{i}, z_{i}\right)=$ $b\left(x_{i}+z_{i}\right)-k\left(x_{i}\right)$, where $b(\cdot)$ is the benefit from energy consumption while $k(\cdot)$ is the cost of investing in renewable energy sources. Naturally, $u_{12}=b^{\prime \prime}-k^{\prime \prime}<0$, so the technology is, in this case, a substitute to extraction. Thus, each user invests strategically little in windmills.

In sum, it does not matter whether $x_{i}$ is first-stage extraction or investments in technology. It does not matter whether the technology is a substitute or a complement to later extraction. As long as $i$ can set $x_{i}$ first, it sets $x_{i}$ such as to commit to more extraction later on. The marginal direct impact on own utility is negligible, but the consequence that other users will extract less is beneficial and of first-order magnitude for $i$. When everyone act in this way, total extraction increases and utilities decline.

\section{Private Resources}

Privatization is both the natural and the celebrated answer to the problem of the commons. Intuitively, a user's incentive to extract is weaker if this user is the residual claimant of the remains. However, privatization may create strategic powers through the resource ownership which, when combined with the dynamic nature of the resource, lead to distortions opposite to those that we have seen for the publicly owned resources. This section discusses market power on the supply side and the demand side before analyzing asymmetric settings with strategic investments in substitute technologies. 


\subsection{Suppliers with Market Power}

We start with a simple symmetric setting. Thus, there are two identical owners ( $i$ and $j$ ) each holding a stock $s_{0}$ of a perfectly storable homogenous good, to be sold in two periods $(t=1,2)$. Productions for $i$ are $x_{i}$ and $y_{i}$ in the first and second periods, respectively. Cumulative productions cannot exceed total availability, $x_{i}+y_{i}=z_{i} \leq s_{0}$. Let $u=u(\cdot)$ denote the periodic consumption utility as a function of the total quantity offered to the market. The market price is $p=u^{\prime}(\cdot)$, or $p=p\left(x_{i}+x_{j}\right)$ and $p\left(y_{i}+y_{j}\right)$ for the two periods, respectively. If producer $i$ sells $x_{i}$ units while $j$ sells $x_{j}$ units in the first period, then $i$ 's profit is $\pi^{1}\left(x_{i}, x_{j}\right)=p\left(x_{i}+x_{j}\right) x_{i}$; similarly, second-period profit is $\pi^{2}\left(y_{i}, y_{j}\right)=p\left(y_{i}+y_{j}\right) y_{i}$. We assume that the profit function is strictly concave. This, in turn, implies that productions of $i$ and $j$ are strategic substitutes: If $j$ produces more, $i$ 's benefit from producing more is reduced (as the price is then lower).

Extraction is costly and, the more one extracts, the deeper into the sea one must drill, or the more remote deposites in the mountain one must mine. We assume that if the cost of extracting $x_{i}$ units is given by $c\left(x_{i}\right)$, then the cost of afterwards extracting $y_{i}$ units is given by the total extraction cost, $c\left(x_{i}+y_{i}\right)$, minus the cost already paid, $c\left(x_{i}\right)$. Over the two periods, $i$ 's payoff is thus:

$$
U_{i}\left(x, y, s_{i}\right)=\pi^{1}\left(x_{i}, x_{j}\right)+\pi^{2}\left(y_{i}, y_{j}\right)-c\left(x_{i}+y_{i}\right) .
$$

We will consider two main situations in oligopolistic resource markets: economic and physical exhaustion of the resource. In the former, the economic environment determines how much of the resource is used in total, whereas in the latter case the total supply will be inelastic.

Economic exhaustion: The first-best requires that marginal utilities are equal across the periods as well as equal to the marginal extraction costs:

$$
\begin{aligned}
u^{\prime}\left(x_{i}+x_{j}\right) & =u^{\prime}\left(y_{i}+y_{j}\right)=c^{\prime}\left(x_{i}+y_{i}\right)=c^{\prime}\left(x_{j}+y_{j}\right) \\
& \Rightarrow x_{i}^{*}+x_{j}^{*}=y_{i}^{*}+y_{j}^{*} .
\end{aligned}
$$

We solve the game by backwards induction. The second-period supply for $i$ and $j$ solve:

$$
\frac{\partial \pi^{2}(\cdot)}{\partial y_{i}}=c^{\prime}\left(x_{i}+y_{i}\right)
$$

reflecting the fact that the equilibrium choices depend on the history of extractions captured by $\left(x_{i}, x_{j}\right)$, that is, $y_{i}=y_{i}\left(x_{i}, x_{j}\right)$. Condition (10) implies that own past

production decreases current equilibrium production, $\frac{\partial y_{i}}{\partial x_{i}}<0$, and also that $\frac{\partial y_{j}}{\partial x_{i}}>0$, because productions are strategic substitutes. Given this, reducing the first-period choice of $x_{i}$ can be seen as a strategic investment: the larger is the $i$ 's stock in the ground (i.e., the lower is $x_{i}$ ) at the beginning of the second period, the tougher is $i$ as a competitor to $j$, and the lower is $j$ 's optimal second-period extraction level.

The equilibrium first-period extraction, $x_{i}$, must satisfy the first-order condition:

$$
\frac{\partial \pi^{1}(\cdot)}{\partial x_{i}}+\frac{\partial \pi^{2}(\cdot)}{\partial y_{j}} \frac{\partial y_{j}}{\partial y_{i}} \frac{\partial y_{i}\left(x_{i}, x_{j}\right)}{\partial x_{i}}=c^{\prime}\left(x_{i}+y_{i}\right)
$$


The second term on the left is the strategic investment effect, which is negative: using the resource today is costly not only because of the extraction cost but also because of the second period market share contest. Due to the concavity profits, firms then produce less in the first period than in the second period: $x_{i}<y_{i}$.

Proposition 4 Under economic exhaustion, too little is extracted relative to the firstbest. Given the extracted amount, too little is extracted in the first period $\left(x_{i}<y_{i}\right)$.

Proof. Comparing (10) and (11),

$$
\frac{\partial \pi^{1}(\cdot)}{\partial x_{i}}>\frac{\partial \pi^{2}(\cdot)}{\partial y_{i}} \Rightarrow x_{i}<y_{i}
$$

Together with (10), result (12) gives for both $i$ and $j$

$$
\begin{aligned}
\frac{\partial \pi^{2}(\cdot)}{\partial y_{i}} & =c^{\prime}\left(x_{i}+y_{i}\right)<u^{\prime}\left(y_{i}+y_{j}\right) \\
& \Rightarrow x_{i}+y_{i}<x_{i}^{*}+y_{i}^{*}
\end{aligned}
$$

where $x_{i}^{*}+y_{i}^{*}$ is the socially optimal extraction satisfying $u^{\prime}\left(x_{i}^{*}+x_{j}^{*}\right)=u^{\prime}\left(y_{i}^{*}+y_{j}^{*}\right)$.

QED.

Physical exhaustion and discounting: The corner-solution $z_{i}=s_{0}$ occurs when the resource in the ground has little or no final value, and when the demand is large enough to absorb the available supply. The resource owners cannot, then, manipulate the overall use in the equilibrium, and the above strategic-investment effects cannot arise. Indeed, when the profits from both periods are the same and $z_{i}=s_{0}$, firms tend to allocate their resource evenly, leading to equalized prices $p_{1}=p_{2}$ in periods $t=1$ and $t=2$, respectively. But time horizons in resource use are typically long, and it would be a gross simplification to ignore discounting, leading to time-differentiated extraction payoffs and distortions in olipolistic resource use even when the total resource use is fixed. If we ignore extraction costs, for now, $i$ 's payoff is:

$$
U_{i}\left(x, y, s_{0}\right)=\pi\left(x_{i}, x_{j}\right)+\delta \pi\left(y_{i}, y_{j}\right),
$$

where $\delta \in(0,1)$ is the discount factor and $x_{i}+y_{i}=s_{0}$ for $i$ and $j$.

The first-best requires that the present-discounted value of the resource is equal across the periods:

$$
\begin{aligned}
u^{\prime}\left(x_{i}+x_{j}\right) & =\delta u^{\prime}\left(y_{i}+y_{j}\right) \Rightarrow \\
x_{i}^{*}+x_{j}^{*} & >y_{i}^{*}+y_{j}^{*} .
\end{aligned}
$$

In equilibrium, the strategic interaction between the oligopolists is effectively a oneshot game, as $y_{i}=s_{0}-x_{i}$ is going to hold under physical exhaustion, implying that interior choices at $t=1$ for every $i$ must satisfy:

$$
\frac{\partial \pi^{1}(\cdot)}{\partial x_{i}}=\delta \frac{\partial \pi^{2}(\cdot)}{\partial y_{i}} .
$$


Comparing (14) and (15) shows that oligopolists follow a quite different allocation principle than the one describing first-best, although the outcomes could potentially coincide. Whether the sellers can intertemporally price discriminate depends on the elasticity of marginal utility, defined as $\epsilon_{M U}(c) \equiv-u^{\prime \prime}(c) c / u^{\prime}(c)>0$ where $c$ is consumption that equals extraction in both periods. The elasticity of demand, at extraction level $c$, is then $\epsilon_{D}(c) \equiv 1 / \epsilon_{M U}(c)$. Firm $i$ that extracts share $\gamma_{i}$ of $c$, has $\epsilon_{D}^{i}(c) \equiv 1 / \gamma_{i} \epsilon_{M U}(c)$.

Proposition 5 Under physical exhaustion and discounting, both oligopolistic and monopolistic resource extraction coincide with the first-best if and only if $\epsilon_{M U}^{\prime}(\cdot)=0$. If $\epsilon_{M U}^{\prime}(\cdot)>0$, too little is extracted in the first period $\left(x_{i}+x_{j}<x_{i}^{*}+x_{j}^{*}\right)$.

Proof: Let $u_{1}$ and $u_{2}$ denote equilibrium utilities in periods 1 and 2, respectively. For symmetric oligopoly allocation, we have $\gamma_{i}^{t}=\gamma_{i}^{t}$ for $t=1,2$, and each $i, j$, and we can consider following:

$$
\begin{aligned}
\frac{\delta u_{2}^{\prime}}{u_{1}^{\prime}} & \leq \frac{\delta \frac{\partial \pi^{2}}{\partial y_{i}}}{\frac{\partial \pi^{1}}{\partial x_{i}}} \Leftrightarrow \frac{u_{1}^{\prime \prime}}{u_{1}^{\prime}} x_{i}+1 \leq \frac{u_{2}^{\prime \prime}}{u_{2}^{\prime}} y_{i}+1 \\
& \Leftrightarrow-\frac{u_{2}^{\prime \prime}}{u_{2}^{\prime}} y_{i} \leq-\frac{u_{1}^{\prime \prime}}{u_{1}^{\prime}} x_{i} \\
& \Leftrightarrow \epsilon_{D}^{1 i} \leq \epsilon_{D}^{2 i} \Leftrightarrow \frac{1}{\gamma_{i}^{1} \epsilon_{M U}^{1}} \leq \frac{1}{\gamma_{i}^{2} \epsilon_{M U}^{2}} \\
& \Leftrightarrow \epsilon_{M U}^{2} \leq \epsilon_{M U}^{1} .
\end{aligned}
$$

As $x_{i}^{*}+x_{j}^{*}>y_{i}^{*}+y_{j}^{*}$, we obtain from (16) and (17) that the planner's and the oligopolists' first-order conditions (14) and (15) coincide if and only if $\epsilon_{M U}^{t}$ is a constant at varied consumption levels:

$$
\frac{\delta u_{2}^{\prime}}{u_{1}^{\prime}}=\frac{\delta \frac{\partial \pi^{2}}{\partial y_{i}}}{\frac{\partial \pi^{1}}{\partial x_{i}}} \Leftrightarrow \epsilon_{M U}^{1}=\epsilon_{M U}^{2} .
$$

On the other hand,

$$
\frac{\delta u_{2}^{\prime}}{u_{1}^{\prime}}=1<\frac{\delta \frac{\partial \pi^{2}}{\partial y_{i}}}{\frac{\partial \pi^{1}}{\partial x_{i}}} \Leftrightarrow \epsilon_{M U}^{2}<\epsilon_{M U}^{1} .
$$

That is, the oligopolists have a higher return for saving the resource to the second period than the planner at the first-best allocation $x_{i}^{*}+x_{j}^{*}>y_{i}^{*}+y_{j}^{*}$ iff $\epsilon_{M U}$ decreases as we move to $t=2$. For the monopoly case, the market share is $\gamma^{t}=1 \mathrm{in}$ both periods. The asymmetric oligopoly case is similar and the proof is omitted. QED

Under constant elasticity of marginal utility, and thus demand, the marginal profit is proportional to price, and therefore the ratio of prices and marginal profits remains constant at all extraction levels: strategic powers do not distort extraction in this case. However, in general, elasticity of demand increases when quantities contract and prices increase; for example, if consumers have a finite choke price at which consumption ceases, $p=u^{\prime}(0)<0$, we have $\epsilon_{D}(0)=\infty$ or $\epsilon_{M U}=0$ and $\epsilon_{D}(\cdot)<\infty$ for positive consumption levels. The marginal profit condition then implies differentiated intertemporal prices, that is, consumers pay more for the resource in the first period than in the second:

$$
\frac{\partial \pi^{1}}{\partial x_{i}}-\delta \frac{\partial \pi^{2}}{\partial y_{i}}=0 \Longrightarrow u_{1}^{\prime}>\delta u_{2}^{\prime}
$$




\subsection{Buyers with Market Power}

The dynamic relationship between resource consumers and suppliers involves strategic decisions also on the buyer side, arising, for example, from the consumer demand management through policies such as consumption taxes, import tariffs and subsidies to alternative technologies, and also from investments in development and adoption of resource substitutes. The policies coordinate the consumer side behavior, and therefore lead to the question of how the consumer side should optimally manage its demand of the resource over time. The key features of this problem are conceptually quite different from those of the oligopolistic setting above: the government (buyer) faces a commitment problem, causing distortions and making the demand management difficult. We illuminate these differences by considering a setting where one single buyer decides on its purchases of the resource from competitive private property resource sellers.

For this, let us assume that there is a continuum of price-taking sellers, each holding a unit of the resource, indexed by $z \in\left[0, s_{0}\right]$. The aggregate cost of extracting the amount $z$ is $c(z)$, as in previous section, and the marginal cost is $c^{\prime}(z)$ which, in equilibrium, must be the price when $z$ units are expected to be purchased. Thus, we can let supply be represented by the function $p(z) \equiv c^{\prime}(z)$, showing the equilibrium price when, in total, $z$ units are bought; we assume that the supply reservation price $p(z)$ is strictly convex. Note that, when there is no discounting, the price must be the same in both periods, and it must be equal the marginal extraction cost when $x+y$ units are extracted. The buyer's pleasure when consuming $x$ units in period one, and $y$ units in period two, is given by $u(x)+u(y)$, where $u(\cdot)$ is assumed to be increasing and strictly concave.

The first-best is thus requiring:

$$
u^{\prime}(x)=u^{\prime}(y)=c^{\prime}(x+y)=p(x+y) \Rightarrow x=y .
$$

In equilibrium, the buyer buys $x$ in the first and $y$ in the second period at price $p(x+y)$, and obtains the payoff:

$$
U(x, y, s)=u(x)+u(y)-(x+y) p(x+y)
$$

where we assume that the buyer's utility is quasi-linear in money. The utility-function is assumed to satisfy $u^{\prime}(0)<p\left(s_{0}\right)$, implying that the resource is economically exhausted (the last unit is too expensive to extract).

Consider, first, the static or normal-form game, where the buyer sets $x$ and $y$ simultaneously in the beginning of the game. In this case, the buyer choses $U_{x}\left(x, y, s_{0}\right)=$ $U_{y}\left(x, y, s_{0}\right)=0$, that is, the marginal consumption utilities are equalized with the marginal purchasing cost for the total amount $x+y=x^{*}+y^{*}$ :

$$
u^{\prime}\left(x^{*}\right)=u^{\prime}\left(y^{*}\right)=p\left(x^{*}+y^{*}\right)+p^{\prime}\left(x^{*}+y^{*}\right)\left(x^{*}+y^{*}\right) .
$$

This is the buyer's commitment solution: the buyer would like to announce consumptions $x^{*}=y^{*}$ satisfying (18) in the first period. Note that the intertemporal allocation is efficient even though too little of the resource is extracted, compared to the first-best.

Consider, next, the extensive form game where the buyer sets $x$ in the first period, unable to commit to $y$, set in the second period. As the second period arrives, the buyer would like choose $y=y^{d}$ satisfying the first-order condition: 


$$
u^{\prime}\left(y^{d}\right)=p\left(x+y^{d}\right)+p^{\prime}\left(x+y^{d}\right) y^{d} .
$$

As this equation is different from (18), it is clear that the ideal policy under commitment is not time consistent. Once the second period arrives, there is no need to pay attention to how a larger $y$ should have raised the price in the first period, and $y$ is thus larger than what the buyer would have preferred to commit to at the beginning of the game.

Proposition 6 Under monopsony, too little is extracted compared to the first-best. Given the extracted amount, too little is extracted in the first period $(x<y)$.

Proof. In equilibrium, the buyer must respect the sellers' arbitrage and thus the first-period price equals the anticipated second-period price,

$$
p_{1}=p(x+y(x)) \text {. }
$$

The f.o.c. for $x$ is:

$$
u^{\prime}(x)+u^{\prime}(y) y^{\prime}-\left(1+y^{\prime}(x)\right) p-(x+y(x))\left(1+y^{\prime}(x)\right) p^{\prime},
$$

where $y^{\prime}=y^{\prime}(x)$, as given by (19). By substituting (19) into (21) and rearranging, we get:

$$
u^{\prime}(x)-u^{\prime}(y)=\left(1+y^{\prime}(x)\right) x p^{\prime}(x+y)>0 .
$$

By differentiating (19), we can verify $-1<y^{\prime}(x)<0$, confirming the inequality in (22). The fact that too little is extracted in total follows since, even in the second period, $u^{\prime}(y)>p,(19)$ shows. $Q E D$

As in the static version of this game, too little is extracted. The buyer buys little in order to keep the price low. In contrast to the static version, i.e., for the case where the buyer can commit, the buyer ends up consuming more in the second period than in the first. Ideally, the buyer would like to commit to consume little in both periods, such that the price would stay low throughout the two periods. Once the second period arrives, however, the price paid for $x$ is sunk and the buyer can raise $y$ without changing that price. Of course, the sellers anticipate that $y$ is going to be large and rational expectations ensure that the price stays the same across both periods.

The comparison illustrates a general phenomenon: first-best policies for the buyer, designed to influence resource consumption over time, are not generally time consistent. We will discuss particular policies such as tariffs on resource imports in a richer setting in Section 4, but the time-inconsistency of tariffs follows directly from the current analysis. To make a sharp case, consider a government regulating consumption using a unit tax $\tau$. The first-best tax, implementing (18), is defined by:

$$
u^{\prime}\left(x^{*}\right)=u^{\prime}\left(y^{*}\right)=p\left(x^{*}+y^{*}\right)+\tau^{*} .
$$

Without commitment, the resource market expects the buyer to deviate from this and set lower taxes in the future, $\tau^{d}<\tau^{*}$. The equilibrium taxes, $\tau_{1}$ and $\tau_{2}$, will implement equilibrium consumptions, as given by (22). Thus, the taxes must decline over time:

$$
u^{\prime}(x)-p=\tau_{1}>u^{\prime}(y)-p=\tau_{2} .
$$


Connections to durable goods and Coase conjecture. It turns out that the resource buyer's commitment problem is conceptually equivalent to that of the durablegood monopoly. Indeed, rather than assuming a strategic resource buyer, consider a monopoly producing and selling a durable-good with zero cost. Buyers have measure $z \in\left[0, s_{0}\right]$ and their reservation price for purchase $p(z)$ is declining in index $z$ which, in this monopoly case, is the consumer stock that is served. Assuming that the monopoly can come to the market twice, selling first $x$ units and then $y$ units while satisfying $x+y \leq s_{0}$, we could follow the steps above to demonstrate the monopoly's commitment problem: the monopoly has incentives to lower prices in the future to serve more demand than initially announced. Anticipating this, consumers will not accept the high prices in the first period, so the monopoly has to serve the customers with a low price in both periods. If the monopoly can frequently interact with the market, the price tends to approach the seller's reservation price. Ronald Coase conjectured that, in such a situation, the monopoly power vanishes "in the twinkling of an eye". The subsequent literature has identified a number of settings where the monopoly can escape the conjecture, including cost-structures that allow commitment to production-smoothing over time and strategies that build reputation for the monopolist. This conceptual connection to durable goods is valuable, since the lessons from the well-explored theory of the durable-good monopoly can be imported to the resource context.

\subsection{Technologies and Strategies}

We will now consider a situation with one buyer, one seller, and a resource that will be physically exhausted. The seller owns the resource stock $s_{0}$ and decides how much to extract in the first period, $y$. The remaining part, $s_{0}-y$, will be extracted in the second period. For simplicity, we assume that there are no extraction costs. The buyer's utility from consumption is $u(\cdot)$, as before. In addition, the buyer decides on $x$, now interpreted as a substitute technology. Assume $x \in[0,1]$ is investment in an R\&D program that produces a substitute for the resource, valued $\bar{v}$, with probability $x$. The investment cost, $C(x)$, is assumed to be increasing and strictly convex in $x$. Both the buyer and the seller has utility functions that are quasi-linear in money. If $w\left(p_{1}\right)=\max _{y} u(y)-p_{1} y$ represents the buyer's indirect utility for period one, given the price $p_{1}$, and similar for period two, then the buyer's total payoff is:

$$
w\left(p_{1}\right)+(1-x) w\left(p_{2}\right)+x \bar{v}-C(x) .
$$

The seller's payoff is

$$
p_{1} y+(1-x) p_{2}\left(s_{0}-y\right) \text {. }
$$

The first-best is given by $x$ and $y$ satisfying the first-order conditions:

$$
\begin{aligned}
u^{\prime}(y) & =(1-x) u^{\prime}\left(s_{0}-y\right), \\
C^{\prime}(x) & =\bar{v}-u\left(s_{0}-y\right) .
\end{aligned}
$$

As above, we simply assume that the second-order conditions hold. We will now contrast the first-best with strategic interaction outcomes where one side has the first-mover advantage. The timing of moves can make a huge difference in the resource use settings, 
and the appropriate approach is not always obvious. For example, if the buyer can first commit to the investment level, then it might be able to extract part of the resource rent. Alternatively, if it is natural that the resource market is active before investments are contemplated, then the seller side can potentially influence and manipulate the buyer's investment to its own advantage. We start with simultaneous moves.

Investment-extraction contest: Assume, first, simultaneous moves: the buyer chooses $x$ and the seller decides on sales $y$ for the first period. It is easy to show that the equilibrium choices must satisfy the first-order conditions:

$$
\begin{aligned}
C^{\prime}(x) & =\bar{v}-w\left(p_{2}\right) \\
\frac{\partial \pi^{1}(\cdot)}{\partial y} & =(1-x) \frac{\partial \pi^{2}(\cdot)}{\partial y},
\end{aligned}
$$

where $\frac{\partial \pi^{1}(\cdot)}{\partial y}=p_{1}+y p_{1}^{\prime}(y)$ is the marginal revenue for period 1 , and $\frac{\partial \pi^{2}(\cdot)}{\partial y}$ similarly for period 2. Interestingly, the seller will not distort sales if there is no investment: $1-x$ takes the role of the discount faction in Section 3.1 where we saw that no discounting leads to first-best extraction. Moreover, we saw that under constant elasticity of marginal utility $\varepsilon_{M U}^{\prime}(\cdot)=0$, the seller side distortion vanishes even for $x>0$.

Proposition 7 Under simultaneous moves, the buyer invests too much in substitute technologies. If $\varepsilon_{M U}^{\prime}(\cdot)>0$, first-period extraction is too conservative, given $x$.

Proof: Since $w\left(p_{2}\right)<u\left(s_{0}-y\right)$, comparing (23) to the first-best reveals that the buyer invests too much, tilting the supply towards the present. The seller's policy follows from Proposition 5. QED

We will now explore how changing the timing of events alter the results.

Strategic investments: Suppose the buyer first chooses $x$. After observing $x$, the seller thereafter allocates the resource optimally according to (24), as before.

The buyer's first-order condition can be written as:

$$
\begin{aligned}
C^{\prime}(x)+\left[\bar{v}-w\left(p_{2}\right)\right] & =v^{\prime}\left(p_{1}\right) \frac{\partial p_{1}}{\partial x}+(1-x) w^{\prime}\left(p_{2}\right) \frac{\partial p_{2}}{\partial x} \\
& =-y \frac{\partial p_{1}}{\partial x}-(1-x)\left(s_{0}-y\right) \frac{\partial p_{2}}{\partial x}
\end{aligned}
$$

where $\frac{\partial p_{1}}{\partial x}=\frac{\partial p}{\partial y} \frac{\partial y}{\partial x}$ and $\frac{\partial p_{2}}{\partial x}=-\frac{\partial p}{\partial\left(s_{0}-y\right)} \frac{\partial y}{\partial x}$.

Proposition 8 Under buyer's leadership, the investment level is the same as with simultaneous moves when $\varepsilon_{M U}^{\prime}(\cdot)=0$. If $\varepsilon_{M U}^{\prime}(\cdot)>0$, the investments are larger under buyer's leadership, and this leads to more first-period conservation, than under simultaneous moves.

Proof: We can rewrite the equilibrium condition as

$$
\begin{aligned}
C^{\prime}(x)-\left[\bar{v}-w\left(p_{2}\right)\right] & =-\left[y \frac{\partial p_{1}}{\partial y}-(1-x)\left(s_{0}-y\right) \frac{\partial p_{2}}{\partial\left(s_{0}-y\right)}\right] \frac{\partial y}{\partial x} \\
& =\left[p_{1}-(1-x) p_{2}\right] \frac{\partial y}{\partial x} \geq 0
\end{aligned}
$$


where the inequality follows from Proposition 5. If $\varepsilon_{M U}^{\prime}(\cdot)=0$, then $p_{1}-(1-x) p_{2}=0$; if $\varepsilon_{M U}^{\prime}(\cdot)>0$, the equality is strict. $Q E D$

Let us then move on to the seller's leadership in the relationship.

Strategic extraction: Consider now the same model with the reversed timing: the seller is first setting supplies, and then the buyer decides on effort $x$. After observing $y$, the buyer takes the future resource scarcity as given, and follows the rule (23), given $w\left(p_{2}\right)$. But $w\left(p_{2}\right)$ is not exogenous: the seller can manipulate the buyer's payoff from effort by optimally choosing $y$ subject to (23).

Proposition 9 Under seller's leadership, first-period extraction is more conservative than under simultaneous moves, since extracting less today reduces the buyer's investment in substitute technology.

Proof: The seller's the first-order condition is

$$
\begin{aligned}
\frac{\partial \pi^{1}(\cdot)}{\partial y}-(1-x) \frac{\partial \pi^{2}(\cdot)}{\partial y} & =x^{\prime}(y) p_{2}\left(s_{0}-y\right)>0 \\
& \Rightarrow p_{1}>(1-x) p_{2}
\end{aligned}
$$

where $x^{\prime}(y)=w^{\prime}\left(p_{2}\right) p^{\prime}\left(s_{0}-y\right) / C^{\prime \prime}(x)>0$ from (23). Thus, even without the seller's price-discrimination option, $\varepsilon_{M U}^{\prime}(\cdot)=0$, the seller over-saves the resource to pre-empt investments. $Q E D$.

\section{Private Inputs and Public Outputs}

\subsection{A model with extraction, emission, and policies}

The two proceeding sections present models that are different, but note that they are not mutually exclusive. In reality, some aspects of the resource may be private, while other are public. Fossil fuel deposits may be owned by individual countries (or companies) but the emission generated when consuming the fuel equals extraction of a public good: clear air. A realistic model should capture both aspects.

This section presents a classic model where the resource is privately owned but extraction generates an externality. One country, or coalition of countries, is harmed by this externality and it can commit to a policy at the beginning of the game. The equilibrium policy will reflect the public as well as the private aspect of the resource in illuminating ways.

First, a policy is set by a climate-coalition, $M$. Second, the producers and consumers in nonparticipating countries decide how much to extract and consume. We abstract from internal conflicts within $M$ and treat $M$ as one player. We will now let $y_{i}$ denote $i$ 's consumption of fossil fuel while $x_{i}$ denotes $i$ 's extracted amount. A country benefits from consuming fuel, but extraction is costly. The extraction costs can, as mentioned above, be captured by the cost $c_{i}\left(x_{i}\right)=-v_{i}\left(s_{i}-x_{i}\right)$. With trade in fossil fuel, $i$ can consume a different amount, $y_{i}$, than what it extracts. If $p$ is the world price for fossil fuel, we assume that the payoff to every nonparticipating country, $i \in N \equiv I \backslash M$, is:

$$
U_{i}=u_{i}\left(y_{i}\right)-c_{i}\left(x_{i}\right)-p\left(y_{i}-x_{i}\right), i \in N .
$$


Assume that the function $u_{i}\left(y_{i}\right)$ is twice differentiable and satisfies $u_{i}^{\prime}>0 \geq u_{i}^{\prime \prime}$. The total consumption of fossil fuel is also equal to extraction of clean air, measured by $s$. For simplicity, assume only $M$ cares about clean air. $M$ 's utility is then

$$
U_{M}=u_{M}\left(y_{M}\right)-c_{M}\left(x_{M}\right)+v\left(s_{0}-\sum_{I} y_{i}\right)-p\left(y_{M}-x_{M}\right), i=M .
$$

In the first stage of the game, $M$ sets environmental policies. This amounts to setting the policy-pair $\left(x_{M}, y_{M}\right)$ if relying on quotas for extraction and consumption, for example. The price for fuel will then adjust to ensure that the market clears:

$$
\sum_{I} y_{i}=\sum_{I} x_{i}
$$

In the second stage of the game, each $i \in N$ maximizes $U_{i}$ by choosing $\left(x_{i}, y_{i}\right)$, taking the price $p$ as given. Thus, by assumption, only $M$ recognizes its effect on the price. Alternatively, we can assume that the individual consumers decide $y_{i}$ and the individual suppliers decide $x_{i}$, and that these take $p$ as given.

\subsection{The Equilibrium Policy}

The first-best is given by equalizing every country's marginal benefit of consumption to the marginal cost of production, plus the marginal environmental harm:

$$
u_{i}^{\prime}\left(y_{i}^{*}\right)=u_{j}^{\prime}\left(y_{j}^{*}\right) \text { and } u_{j}^{\prime}\left(y_{j}^{*}\right)=c_{j}^{\prime}\left(x_{i}^{*}\right)-v^{\prime}\left(s_{0}-\sum_{I} y_{i}^{*}\right) \forall i, j \in I .
$$

In equilibrium, however, at the second stage, nonparticipating countries consume according to:

$$
u_{i}^{\prime}\left(y_{i}\right)=p \Rightarrow y_{i}=D_{i}(p) \equiv u_{i}^{\prime-1}(p) .
$$

The demand by $i \in N$ is thus given by $D_{i}(p)$. A country's extraction level satisfies:

$$
p=c_{i}^{\prime}\left(x_{i}\right) \Rightarrow x_{i}=S_{i}(p) \equiv c_{i}^{-1}(p) \forall i \in N .
$$

At the first stage, $M$ sets its policy such as to maximize $U_{M}$, taking into account that the market must clear at the second stage, i.e.:

$$
\begin{aligned}
I \equiv y_{M}-x_{M} & =S(p)-D(p), \text { where } \\
S(p) & \equiv \sum_{N} S_{i}(p), \\
D(p) & \equiv \sum_{N} D_{i}(p) .
\end{aligned}
$$

$M$ must take (27)-(29) into account and, by differentiating these equations, we can learn how $M$ 's policy affects the world price and nonparticipants' behavior:

$$
\left\{\begin{array}{c}
d x_{i}=S_{i}^{\prime}(p) d p \forall i \in N \\
d y_{i}=D_{i}^{\prime}(p) d p \forall i \in N \\
d x_{M}-d y_{M}=\sum_{N}\left(d y_{i}-d x_{i}\right)
\end{array}\right\} \Rightarrow\left\{\begin{array}{c}
\frac{d p}{d x_{M}-d y_{M}}=\frac{1}{S^{\prime}(p)-D^{\prime}(p)} \\
\frac{d x_{i}}{d x_{M}-d y_{M}}=\frac{S_{i}^{\prime}(p)}{S^{\prime}(p)-D^{\prime}(p)} \\
\frac{d y_{i}}{d x_{M}-d y_{M}}=\frac{D_{i}^{\prime}(p)}{S^{\prime}(p)-D^{\prime}(p)}
\end{array}\right\}
$$


Given these constraints, it is straightforward to derive $M$ 's equilibrium policy at the first stage:

Proposition 10 At the first stage, $M$ 's equilibrium policy will satisfy:

$$
\begin{gathered}
\left(\frac{S^{\prime}(p)}{S^{\prime}(p)-D^{\prime}(p)}\right) v^{\prime}+\frac{y_{M}-x_{M}}{S^{\prime}(p)-D^{\prime}(p)}=u_{M}^{\prime}\left(y_{M}\right)-p, \\
\left(1-\frac{S^{\prime}(p)}{S^{\prime}(p)-D^{\prime}(p)}\right) v^{\prime}-\frac{y_{M}-x_{M}}{S^{\prime}(p)-D^{\prime}(p)}=p-c_{M}^{\prime}\left(x_{M}\right) .
\end{gathered}
$$

If $M$ implements its policy using taxes on domestic consumption and extraction, these must be equal to the left-hand sides of (30)-(31):

$$
\begin{aligned}
\tau_{y} & =\left(\frac{S^{\prime}(p)}{S^{\prime}(p)-D^{\prime}(p)}\right) v^{\prime}+\frac{y_{M}-x_{M}}{S^{\prime}(p)-D^{\prime}(p)} \\
\tau_{x} & =\left(1-\frac{S^{\prime}(p)}{S^{\prime}(p)-D^{\prime}(p)}\right) v^{\prime}-\frac{y_{M}-x_{M}}{S^{\prime}(p)-D^{\prime}(p)} .
\end{aligned}
$$

It is interesting to disenthangle the different effects determining the equilibrium taxes.

First, isolate the private aspect of the resource by abstracting from the negative externality. When $v^{\prime}=0$, the first terms on the right-hand side vanishes. In this case, $M$ prefers to tax/subsidize its own extraction and consumption only because this affects its terms of trade. If $M$ is importing fossil fuel, $y_{M}>x_{M}, M$ prefers to subsidize extraction and tax consumption, since committing to such policies reduce the world price of fossil fuel. By committing to consume little, for example, the price declines, benefitting the importer. This incentive is somewhat similar to lessons from the previous section with market power. If $M$ is exporting, $M$ would prefer to subsidize consumption and tax extraction in order to raise the equilibrium price.

Second, isolate the negative externality by assuming that $M$ is consuming approximately the same amount which it extracts, such that $y_{M} \approx x_{M}$. Then, $M$ prefers a tax on both consumption and extraction. The magnitude of each tax depends on the elasticity of supply and demand in the nonparticipating countries. Suppose supply is quite inelastic ( $S^{\prime}$ is small) while demand is elastic ( $D^{\prime}$ is large). Then, if $M$ tries to reduce its consumption, $p$ drops and the other countries will just consume more. This creates carbon leakage on the demand side. Given this carbon leakage, $M$ is better of taxing and reducing its supply rather than its consumption. After all, global consumption must equal global supply. Suppose, instead, supply is very elastic ( $S^{\prime}$ is large) while demand is inelastic ( $D^{\prime}$ is small). Then, if $M$ tries to reduce its supply, $p$ increases and the other countries will just extract more. This creates a carbon leakage on the supply side, and $M$ is better of by focusing on reducing and taxing its consumption rather than its supply.

Interestingly, note that the coalition sets taxes such that the sum of the taxes is always equal to the Pigouvian tax, $v^{\prime}$. Furthermore, when setting the optimal taxes, the effects of the public-good aspect (the negative externality) and the private aspect (the concern for $M$ 's terms-of-trade) are simply additive. Unfortunately, the outcome if far from first-best: Neither marginal benefits nor marginal costs are equalized. The nonparticipants typically consume too much and extract too much. The composition of 
the taxes are driven by both the concern for carbon leakage as well as $M$ 's concern for its terms-of-trade, and this distorts trade.

No matter the choice among these tax instruments, this equilibrium is highly inefficient: marginal extraction costs are not equalized (nonparticipants tend to extract too much), marginal benefits are not equalized (nonparticipants tend to consume too much), and the first-stage policy is set such as to improve the coalition's terms-of-trade. However, since the countries can trade fossil fuels, one may argue that they might also be able to trade the fossil fuel deposits. If so, the coalition $M$ may want to purchase deposits in the nonparticipating countries, to prevent them extracting more as soon as $M$ decides to extract less. Such trade may take place in equilibrium and be highly beneficial in the present model, it can be shown.

\section{Conclusions}

Game theory is the natural tool for analyzing the strategic use of natural resources. In this article, we have been interested in private as well as public resources, static as well as dynamic common pool problems, technologies that can reduce the need to extract or make extraction cheaper. Although we have employed a very simple two-stage game throughout the article, we found that it could generate a number of important lessons:

1. In the static common-pool problem, where the resource is publicly owned, each user extracts too much.

2. In a dynamic common-pool problem, each user tries to discourage the other users from extracting in the future. This is achieved, in equilibrium, by inefficiently extracting a lot early on, and by investing heavily in complementary technologies (such as extraction technology) but strategically little in green technologies. In all these cases, the dynamic common-pool problem is worse than the static common-pool problem.

3. If the resource is privately owned, instead, the results are reversed: Whether the buyer or the sellers have market power, extraction rates tend to be too small. Furthermore, the intertemporal allocation of extraction tends to be too conservative (too little is extracted in the beginning), since each seller would like to commit to extract more in the future to discourage the competitors from extracting then, or to discourage the buyer from investing in substitute technology.

4. The above insights are combined if the resource has some aspect that is private (e.g., the right to extract) and other aspects that are public (e.g., the environmental harm). The party that is affected by the externality prefers to commit to a policy mitigating it. In equilibrium, however, the policy will be set such as to improve the party's terms-of-trade as well as the environment.

The lessons from these simple games are quite negative, but the following nonexhaustive reading-list is also including a few articles discussing solutions. The world is still imperfect, so the reader is encouraged to contribute to the list in their future research. 


\section{Further Reading}

Beccherle, Julien and Tirole, Jean (2011): "Regional Initiatives and the Cost of Delaying Binding Climate Change Agreements," Journal of Public Economics, Volume 95, Issues 11-12, Pages 1339-1348.

Bergstrom, T. C. 1982. "On Capturing Oil Rents with a National Excise Tax", American Economic Review 71, 194-201.

Buchholz, Wolfgang and Konrad, Kai (1994): "Global Environmental Problems and the Strategic Choice of Technology," Journal of Economics 60 (3): 299-321.

Coase, Ronald H. (1960): "The Problem of Social Cost," Journal of Law and Economics 3: $1-44$.

Dasgupta, P., R. Gilbert, and J. Stiglitz (1983), Strategic Considerations in Invention and Innovation: The Case of Natural Resources, Econometrica 51, 1439-1448.

Dasgupta, P., and G. Heal (1980): Economic Theory and Exhaustible Resources, Cambridge University Press.

Dutta, Prajit K. and Radner, Roy (2006): "A Game-Theoretic Approach to Global Warming," Advances in Mathematical Economics 8: 135-53.

Fudenberg, Drew and Tirole, Jean (1991): Game Theory. MIT Press.

Gerlagh, Reyer, and Liski, Matti (2011): "Strategic resource dependence," Journal of Economic Theory, Volume 146, Issue 2, Pages 699-727.

Golombek, Rolf, and Hoel, Michael (2004): "Unilateral Emission Reductions and CrossCountry Technology Spillovers." Advances in Economic Analysis \& Policy 4 (2), Article 3.

Harstad, Bård (2012): "Climate Contracts: A Game of Emissions, Investments, Negotiations, and Renegotiations," Review of Economic Studies, forthcoming.

Harstad, Bård (2012): "Buy Coal! A Case for Supply-Side Environmental Policy," Journal of Political Economy, 120 (1), 2012: 77-115.

Hoel, Michael (1994): "Efficient Climate Policy in the Presence of Free Riders," Journal of Environmental Economics and Management 27 (3): 259-74.

Hörner, J., and M. Kamien. 2004. "Coase and Hotelling: A Meeting of the Minds", Journal of Political Economy 112, 718-723.

Karp, L., and D. Newbery. 1993. "Intertemporal Consistency Issues in Depletable Resources", In Handbook of Natural Resource and Energy Economics, vol. 3, edited by Allen V. Kneese and James L. Sweeney. Amsterdam: Elsevier Science. 
Levhari, David and Mirman, Leonard J. (1980): "The Great Fish War: An Example Using a Dynamic Cournot-Nash Solution," Bell Journal of Economics 11 (1): 32234 .

Liski, Matti, and Montero, Juan-Pablo (2011): "Market power in an exhaustible resource market: The case of storable pollution permits," The Economic Journal, 121: 116-144.

Markusen, James R. (1975): "International externalities and optimal tax structures," Journal of International Economics 5 (1): 15-29.

Maskin, E., and D. Newbery. 1990. "Disadvantageous Oil Tariffs and Dynamic Consistency ", American Economic Review 80, 143-156.

Ploeg, Frederick van der and de Zeeuw, Art (1992): "International aspects of pollution control," Environmental and Resource Economics 2 (2): 117-39.

Reinganum, J. F., and N.L. Stokey (1985), Oligopoly Extraction of a Common Property Natural Resource: The Importance of the Period of Commitment in Dynamic Games, International Economic Review 26, 161-173.

Van Long, Ngo (2012): "Differential Games and Resources," chapter 148 in the Encyclopedia of Energy, Natural Resource and Environmental Economics. 